\title{
REGISTRO MONETARIO DE LA «VILLA ROMANA» DE HUERTA DE SAN NICOLÁS: APUNTES PARA LA HISTORIA ROMANA DE ÁVILA
}

\author{
MONETARY REGISTRY IN THE "ROMAN VILLA" OF HUERTA DE SAN NICOLÁS: \\ NOTES FOR THE ROMAN HISTORY OF ÁVILA
}

\author{
NOÉ CONEJO DELGADO
}

\section{INTRODUCCIÓN}

En los trabajos de documentación arqueológica realizados en 2002 por Raul Maqueda García - Morales $^{1}$ en la zona abulense de Huerta de San Nicolás, fueron registradas una serie de evidencias de época romana que podrían identificarse con una villa suburbana de la antigua ciudad de Obula, actual Ávila.

Esta intervención arqueológica fue promovida y contratada por el Ayuntamiento de Ávila en el proceso previo de urbanizar el espacio geográfico denominado Plan Parcial ARUP 1/1 San Nicolás 1. Esta zona, según se ha podido extraer de la memoria de intervención conservada en el Museo Arqueológico Provincial de Ávila (Máqueda García-Morales, 2002), está plagada de restos de estructuras hidráulicas que fueron levantadas y explotadas por población mudéjar y morisca, encontrándose también cerca los restos de una necrópolis musulmana conocida con el nombre de Pared de Osos, por lo que era más que evidente la necesidad de un control arqueológico.

Los principales objetivos de la actuación consistían en realizar un prospección visual de las parcelas afectadas por el proceso de urbanización, con tal de identificar los elementos más significativos, conocer las evidencias del posible poblamiento medieval del

\footnotetext{
1. Aprovechamos esta nota para dar las gracias a María Mariné, Directora del Museo de Ávila, y a su personal, por el trato recibido en nuestra visita al Museo en diciembre de 2014. También agradecemos a Raul Maqueda García - Morales su consentimiento para la realización de este trabajo, para la consulta de su memoria y para la utilización y publicación de alguno de los datos que vamos a tratar en las siguientes páginas. Aunque no hemos dispuesto de una documentación completa (imágenes de hallazgos y planos) creemos que con las descripciones que se aportan y los datos presentados se puede hacer una lectura global de la Villa de San Nicolás y poner en relación los hallazgos numismáticos documentados con el devenir socioeconómico de este enclave y el de la ciudad de Ávila.
}

lugar a través de pequeños sondeos, excavación de sondeos y zanjas para poder definir mejor los posibles hallazgos y excavación en superficie de estos si los hubiera.

El espacio delimitado comprendía una extensión aproximada de 7 ha en la desembocadura del Río Chico en el Río Adaja, en la zona SO de la ciudad de Ávila y muy cercano a la Iglesia de San Nicolás, edificio que da nombre a toda una zona de pequeñas propiedades privadas dedicadas hasta hace relativamente poco tiempo a huerta. Los trabajos se englobaron en dos amplios sectores: Pradera I y Pradera II. En el primer ámbito se documentaron un gran número de norias, acequias, y demás estructuras hidráulicas relacionadas con las explotaciones agrícolas características de regadío. En el segundo, además de estructuras similares a las anteriores, también se identificaron niveles de época romana que debido a su singularidad decidieron ser excavados en extensión, descubriéndose los restos de una villa romana con una vida que oscila entre el siglo I y el II d.C.; cuyas evidencias se encontraban en el actual triángulo formado por las calles Alí Caro, Mancebo de Arévalo y Travesía de la Mina (Fig. 1).

\section{LA «VILLA ROMANA» DE SAN NICOLÁS}

De las seis zanjas/sondeos abiertos para poder definir mejor los diferentes hallazgos materiales documentados en la zona de Pradera II, en cuatro se identificaron restos romanos de época alto imperial fechables entre la segunda mitad del siglo I d.C. y finales del siglo II d.C. Observándose que en todas las zanjas se encontraba una unidad estratigráfica similar, se decidió realizar una excavación en superficie con tal de poder averiguar la entidad de los restos.

Así, se pudieron identificar una serie de elementos que demostraban la existencia de un poblamiento romano realizado en dos momentos no muy distantes en el tiempo. En la unidad estratigráfica más antigua (UE 22) se exhumaron estructuras murarías de grandes 


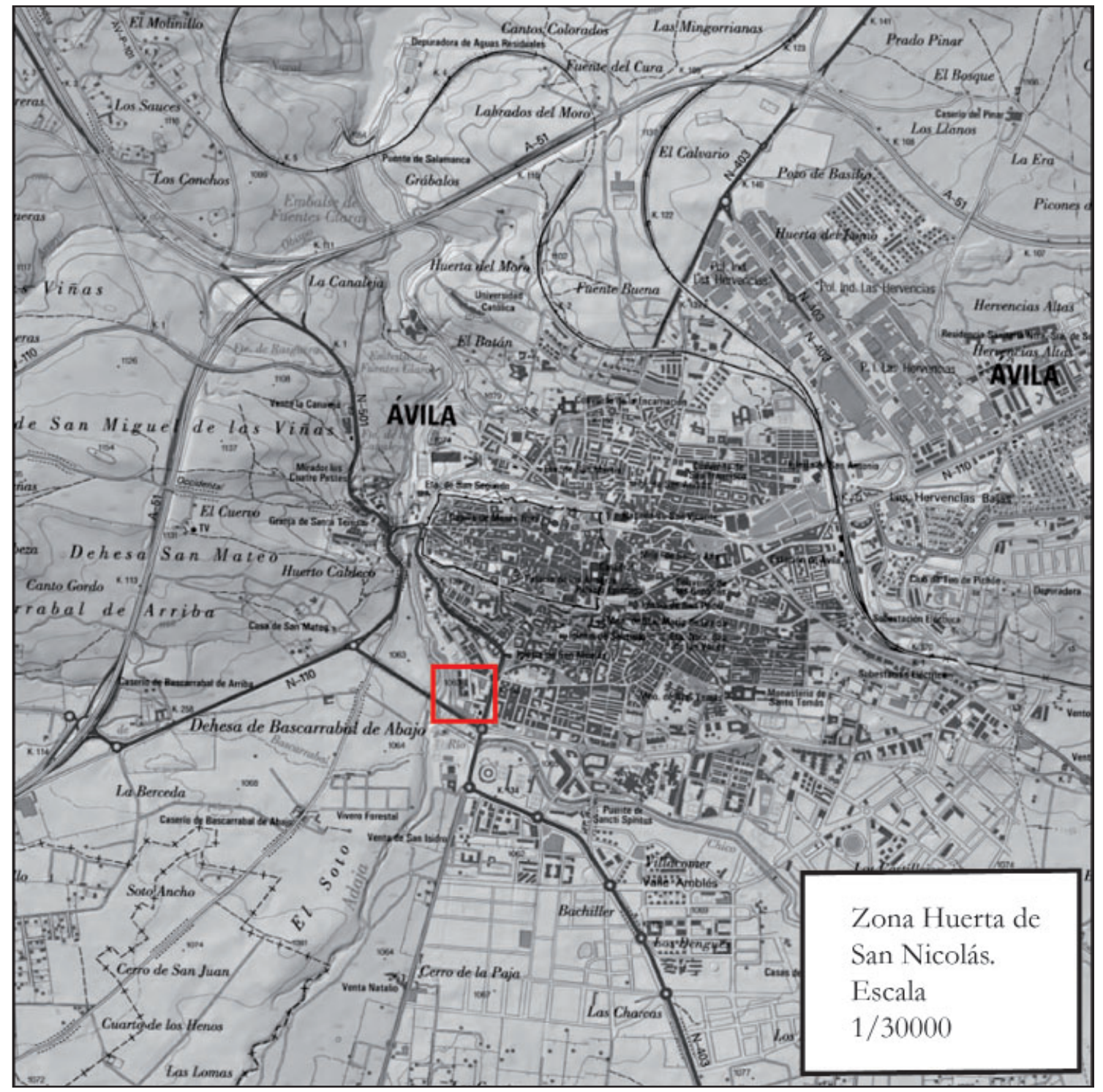

Figura 1: Mapa de Ávila con la situación de la Villa Suburbana de San Nicolás.

dimensiones, levantadas en opus incertum y con una anchura aproximada de $70 \mathrm{~cm}$ en toda su longitud. A su vez, se observaron arranques que permiten pensar en la articulación de un espacio interior de una manera ordenada y preconcebida, según pudieron demostrar los arqueólogos al ver una separación exacta de seis metros entre uno y otro arranque, identificándose por tanto un total de tres posibles habitaciones. Este muro se encontraba asociado a pocos materiales cerámicos como cerámica común engobada de color negro, adscritas al periodo Julio - Claudio, muy parecidas a las encontradas en el yacimiento de Mercado Grande, en el interior de la ciudad de Ávila (Centeno y Quintana, 2005). Como es evidente, estos restos corresponden con los primeros momentos del asentamiento, el cual se abandona sin saber por qué a finales del siglo I d.C., como demuestra un nivel de abandono y amortización (UE 21) asociado a la estructura anteriormente descrita y sin ninguna prueba que indique algún hecho fortuito.
En un nivel superior, perteneciente a la segunda fase del yacimiento, se encuentran algunas estructuras que aprovechan las anteriores de cimiento pero con un aparejo mucho menos cuidado, sin haberse documentado restos de posibles pavimentos, por lo que se puede observar una ocupación breve pero intensa (UE 16) por la gran cantidad de restos materiales asociados, los cuales presentan fechas relativas a la transición del siglo I al II d.C. como son la terra sigillata hispánica documentada con formas tan características de la Drag.15/17, Drag. 24/25, Drag. 36, Drag. 37a, Drag. 29/37 y Drag. 44; algunos fragmentos de paredes finas y cerámica estampillada, y casi la totalidad de las monedas que catalogaremos a continuación.

Como se puede contemplar, no se han exhumado numerosos restos arquitectónicos como para poder identificar en esta ocupación una villa romana tal como conocemos, siendo este hecho el que explica por qué el título de este trabajo tiene entrecomillado estas mismas palabras. Raúl Maqueda también se 
cuestionó en un primer momento si verdaderamente se encontraba ante una villa romana, pero como el mismo arqueólogo justifica en la Memoria ya citada, la lectura de los restos en conjunto permite establecer tal asignación. En primer lugar no hay que olvidar que la zona sufrió muchas alteraciones en época medieval y moderna, por lo que la mayoría de los muros desaparecieron en dichas actividades, dejando prueba de ello un gran número de zanjas y grandes hoyos colmatados con materiales modernos, producto principalmente del expolio de materiales. En segundo lugar, la no identificación de espacios de residencia o áreas productivas no quiere decir que no existieran tales ámbitos, pues la presencia de cerámicas comunes o de semi-lujo como las terra sigillata no solo demuestran un consumo y una actividad comercial, sino también un espacio habitado y de uso doméstico. Por otra parte, Maqueda identifica los elementos definitorios de villa según Columela (Lib. I. Caps. III, IV y V) en este mismo enclave: cercanía a zonas favorables a la actividad agraria y vías de comunicación, una orientación al sur de las estructuras, lo que favorecería la ventilación de la villa: refrigeración en verano y calentamiento en inverno y fuentes de agua corriente. La situación en uno de los márgenes del rio Adaja no será novedad, pues todo su cauce hacia el sur se encuentra plagado de este tipo de yacimientos, aunque de una cronología posterior, pero que indican perfectamente el típico patrón de asentamiento romano de cercanía a fuentes de agua corriente y campos fértiles para el cultivo.

Por lo que respecta al abandono, el propio arqueólogo fue testigo de cómo durante el proceso de excavación el lugar fue anegado por las crecidas del Río Adaja, por lo que pudiera ser esta una de las razones que expliquen la movilidad de los habitantes de esta villa a otro lugar, pero tampoco se han documentado pruebas de hechos fortuitos, por lo que siguen existiendo muchas incógnitas ante esta dejadez a finales del siglo II d.C.

\subsection{REGISTRO MONETARIO Y CATÁLOGO}

Durante la intervención aparecieron un total de 25 monedas, principalmente en el estrato que atestiguaba una segunda ocupación de la villa (UE 16). A su vez, de ese total, solo 15 han podido ser catalogadas y estudiadas al presentar una conservación favorable para su lectura, las 10 restantes se encuentran totalmente frustras impidiendo su identificación.

La muestra corresponde a un hallazgo numismático de tipo acumulativo, es decir, monedas que se han ido perdiendo a lo largo del tiempo en un mismo lugar y que denota su uso corriente por ser moneda fraccionaria. Durante los trabajos de excavación no se anotó sobre la planimetría del yacimiento el lugar exacto de aparición, pero sí sabemos el número de sondeo donde fueron halladas cada una. Al encontrarse monedas de similares cronologías en todos los sondeos que contienen la UE 16, que es el estrato que más moneda ha aportado, el estudio de todas las monedas nos permite una visión global y aproximada de la circulación monetaria del momento en este yacimiento.

La mayor parte de estas piezas se encuentran hoy expuestas en una vitrina monográfica del Museo Provincial de Ávila dedicada a la villa de Huerta de San Nicolás.

A continuación llevaremos a cabo la descripción de las piezas, ordenadas cronológicamente desde las más antiguas a las más modernas y de los valores más altos a los más bajos. Cada pieza será descrita por los criterios convencionales usados en numismática. Será enunciada por su metal (Ae = bronce, oricalco; $\mathrm{Ar}=$ plata), seguida de su valor, del emperador que la acuñó, de su ceca, de la fecha de emisión, del número de UE al que pertenece, junto al de cuadrícula o sondeo y finalmente el numero con el que es identificada la moneda en la memoria de intervención. De algunas monedas no disponemos del número de cuadricula, es probable que existiese un lapsus a la hora de su documentación, no obstante sí sabemos la UE en la que fueron halladas, por lo que no se ve alterada significativamente la lectura del conjunto. Después se efectuará la descripción de su anverso (A.) y reverso (R.), seguida del peso en gramos (g), su módulo en milímetros (mm) y el eje de cuños en el sentido de las agujas del reloj (h). Finaliza la descripción con una referencia bibliográfica. Las abreviaturas utilizadas en esta última parte serán referidas en el punto destinado a la Bibliografía. Finaliza la descripción con el número de inventario del Museo. Las monedas que aparecen como frustro presentan el anverso y el reverso totalmente ilegible, de ahí que creamos conveniente no reproducirlas fotográficamente, otorgándoles una cronología estimada en función de su peso, módulo y grosor. ste tipo de piezas, se ha optado por datarlas con una cronología genérica que engloba el mandato de su emisor.

1.-Ae, As, Claudio I, Roma, 41.54 d.C.; UE. 16, MAQUEDA, 2002, n ${ }^{\circ} 1$.

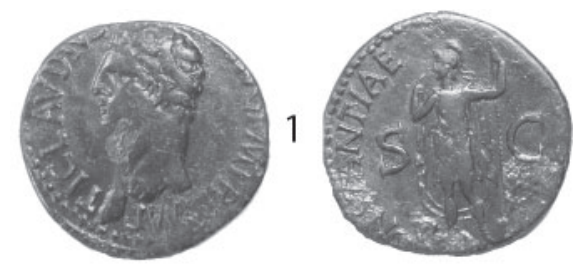

Figura 2: Moneda $\mathrm{n}^{\circ} 1$.

\footnotetext{
A: TI CLAVDIVS CAESAR AVG PM TR P IMP PP. Cabeza desnuda a izquierda.

R: CONSTANTIAE - AVGVSTI. Constancia, con traje militar a izquierda, brazo derecho levantado, con el izquierdo porta una lanza. en el campo SC.
} 
8,88 g; 28 mm; 6 h. Ref. Bibliográfica: RIC I no 95 pág. 127 .

No Inv. 02/79/general/16/1.

2.- Ae, As, Nerón, Roma, 54 - 68 d.C.; UE. 16, MAQUEDA, 2002, nº 4 .

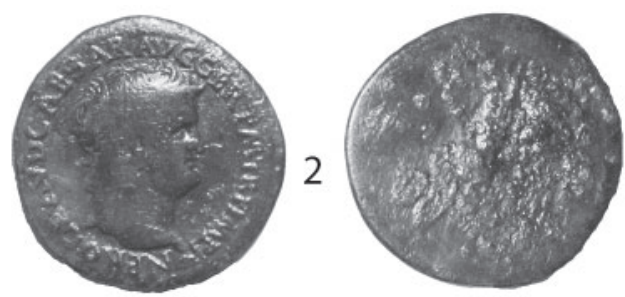

Figura 3: Moneda $\mathrm{n}^{\circ} 2$.

A: NERO CLAVDIVS CAESAR AVG GARM P M TR P IMP PP. Cabeza desnuda a derecha.

R: Frustro

$10,21 \mathrm{~g} ; 29 \mathrm{~mm} ; 6 \mathrm{~h}$.

$\mathrm{N}^{\mathrm{o}}$ Inv. 02/79/general/16/4.

3.- Ae, Dupondio, Vespasiano, Lugdunum, 72 - 73 d.C.; UE. 16, Cuadrícula 2; MAQUEDA, 2002, nº 13.
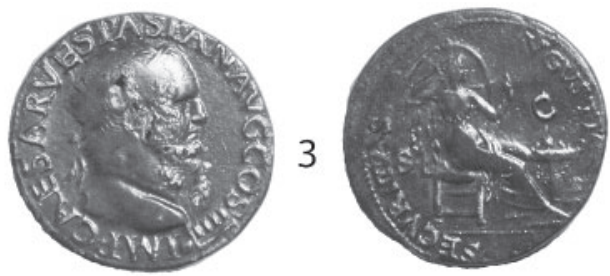

Figura 4: Moneda $\mathrm{n}^{\mathrm{o}} 3$.

A: IMP CAESAR VESPASIAN AVG COS IIII. Cabeza a derecha, radiada, con globo debajo del cuello. R: SECVRITAS AVGVSTI. Seguridad sentada a derecha, la cabeza le descansa en su brazo, porta cetro y delante de ella hay un altar con una antorcha. S - C en campo.

13,80 g; 28 mm; 9h. Ref. Bibliográfica: RIC II n 744 pág. 102.

$\mathrm{N}^{\mathrm{o}}$ Inv. 02/79/C-2/16/13.

4.- Ae, Dupondio, Vespasiano, Roma, 74 d.C.; UE. 16, Cuadrícula 4; MAQUEDA, 2002 nº 16.
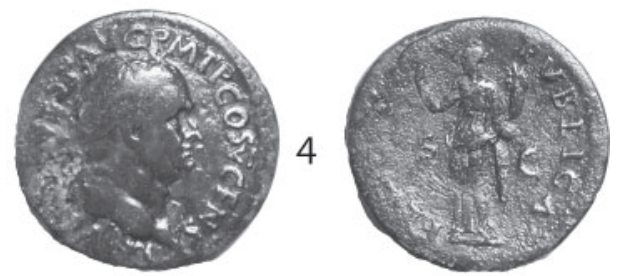

Figura 5: Moneda $n^{\circ} 4$.
A: IMP CAES VESP AVG P M T P COS V CENS. Cabeza radiada a derecha.

R: FELICITAS PUBLICA. Felicidad a izquierda, porta caduceo y cornucopia. S - C en campo.

9,44 g; 28 mm; 6h. Ref. Bibliográfica: RIC II n 554 pág. 80 .

$\mathrm{N}^{\mathrm{o}}$ Inv. 02/79/C-4/16/16.

5.- Ae, Dupondio, Vespasiano, Lugdunum, 77 - 78 d.C.; UE. 16, MAQUEDA, 2002, $\mathrm{n}^{\circ} 6$.
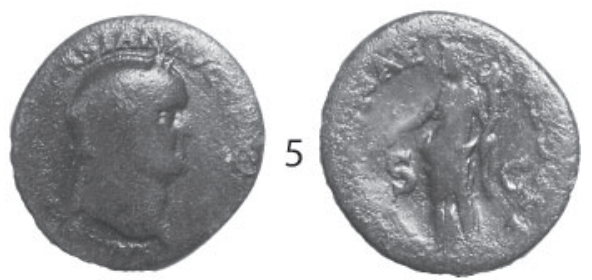

Figura 6: Moneda $\mathrm{n}^{\mathrm{o}} 5$.

A: IMP CAES VESPASIAN AVG P M TR PPP CS VIII. Cabeza laureada a derecha, globo debajo del cuello.

R: FORTVNAE REDVCIS. Fortuna a izquierda, porta timón sobre globo y cornucopia. $\mathrm{S}$ - $\mathrm{C}$ en campo. 9,65 g; 28 mm; 9 h. Ref. Bibliográfica: RIC II no 754b pág. 103.

$\mathrm{N}^{\mathrm{O}}$ Inv. 02/79/general/16/6.

6.- Ae, As, Vespasiano, Roma, 71 d.C.; UE. 22, Cuadrícula 5; MAQUEDA, 2002, nº 19.
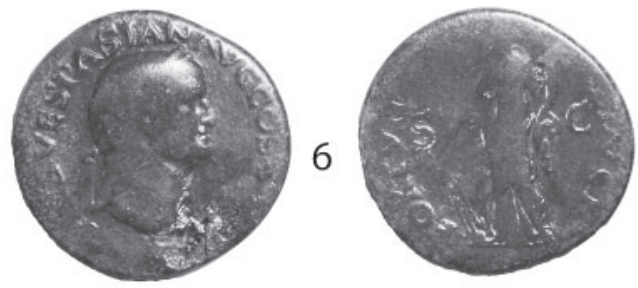

Figura 7: Moneda $\mathrm{n}^{\mathrm{o}} 6$.

A: IMP CAES VESPASIAN AVG COS III. Cabeza laureada a derecha, globo debajo del cuello.

R: FORTUNAE REDVCI. Fortuna a izquierda, porta timón sobre globo y cornucopia, $\mathrm{S}-\mathrm{C}$ en campo.

8,69 g; 29 mm; 11 h. Ref. Bibliográfica: RIC II n 487 pág 73 .

No Inv. 02/79/C-5/22/19.

7.--Ae, As, Vespasiano, Roma, 74 d.C.; UE. 16, Cuadrícula 4; MAQUEDA, 2002, nº 15.

A: IMP CAES VESP AVG P M T P COS V CENS. Cabeza laureada a derecha.

R: PAX AVGVST. Paz a izquierda, apoyanda sobre ara, porta caduceo y ramo. $\mathrm{S}-\mathrm{C}$ en campo. 


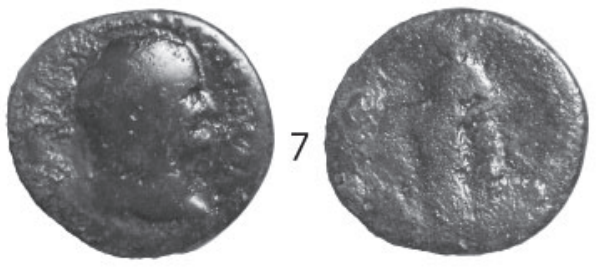

Figura 8: Moneda $n^{0} 7$.

$8,45 \mathrm{~g} ; 27$ mm; 7 h. Ref. Bibliográfica: RIC II nº 559a pág. 80 .

No Inv. 02/79/C-4/16/15.

8.- Ae, As, Vespasiano, 69 - 79 d.C.; UE. 16, Cuadrícula 1; MAQUEDA, 2002, nº 11 .
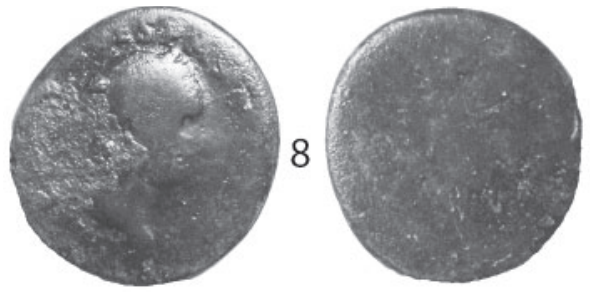

Figura 9: Moneda $\mathrm{n}^{\circ} 8$.

A: ... VESPAS... Cabeza laureada a derecha.

R: Frustro

9,26 g; $29 \mathrm{~mm} ;-\mathrm{h}$.

$\mathrm{N}^{\mathrm{o}}$ Inv. 02/79/C-1/16/11.

9.- Ae, As, Domiciano, Roma, 85 d.C.; UE. 16, MAQUEDA, 2002, nº 5 .
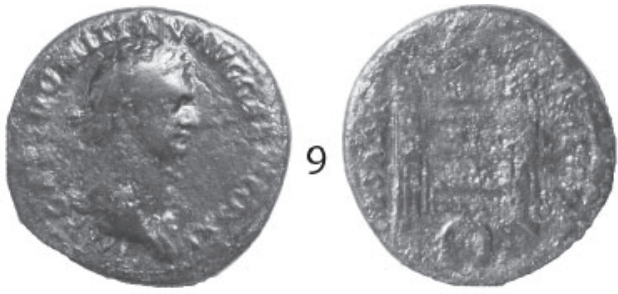

Figura 10: Moneda ${ }^{\circ} 9$.

A: IMP CAES DOMITIAN AVG GERM COS XI. Cabeza laureada a derecha con aegis sobre el hombro. R: SALVTI - AVGVSTI. Altar. S - C en campo. 10,51g.; 29 mm; 9h. Ref. Bibliográfica: RIC II no 272 pág. 188.

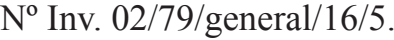

10.-Ar, Denario, Trajano, Roma, 101 - 102 d.C.; UE. 16, Cuadrícula 1; MAQUEDA, 2002, nº 7.

A: IMP CAES NERVA TRAIANVS AVG GERM Busto laureado a derecha.

R: P M TR P COS IIII P P. Victoria, de frente a derecha, porta corona y palma.

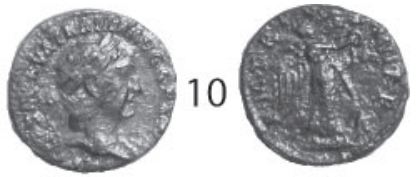

Figura 11: Moneda $\mathrm{n}^{\mathrm{o}} 10$.

3,30 g; 18 mm; 7h. Ref. Bibliográfica: RIC II no 59 pág. 248.

$\mathrm{N}^{\mathrm{o}}$ Inv. $02 / 79 / \mathrm{C}-1 / 16 / 7$.

11.-Ar, Denario, Trajano, Roma, $114-117$ d.C.; UE. 16, MAQUEDA, 2002, nº 8 .
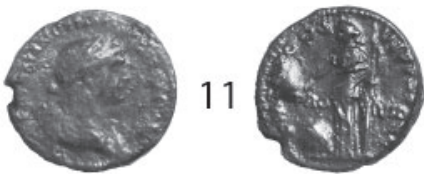

Figura 12: Moneda $\mathrm{n}^{\mathrm{o}} 11$

A: IMP CAES NER TRAIAN OPTIM AVG GER DAC PARTHICO. Busto laureado a derecha con coraza.

R: PARTHICO P M TR P COS VI P P S P Q R. Providencia a izquierda portando lanza, globo en el suelo. PRO - VID en campo.

3,26 g; 17 mm; 11 h. Ref. Bibliográfica: RIC II nº 363 pág. 269.

$\mathrm{N}^{\circ}$ Inv. 02/79/general/16/8.

12.- Ae, Dupondio, Trajano, Roma, 98 - 99 d.C.; UE. 16, MAQUEDA, 2002, nº 9.
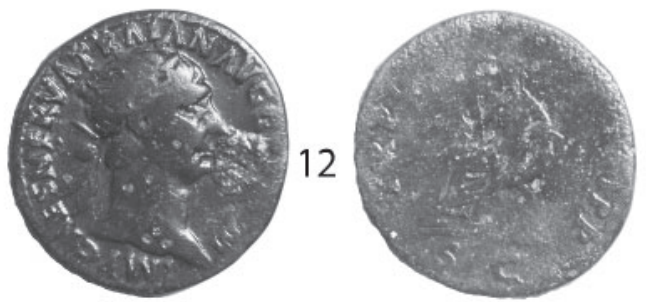

Figura 13: Moneda $\mathrm{n}^{\mathrm{o}} 12$.

A: IMP CAES NERVA TRIAN AVG GERM P M. Cabeza radiada a derecha.

R: TR POT COS II PP. Abundancia a izquierda, sentada en silla formada por dos cornucopias, porta cetro. $\mathrm{S}-\mathrm{C}$ en el campo.

10,64 g; 29 mm; 10 h. Ref. Bibliográfica: RIC II n 398 pág. 273.

$\mathrm{N}^{\mathrm{o}}$ Inv. 02/79/general/16/9.

13.- Ae, Dupondio, Adriano, Roma, 134 - 138 d.C.; UE. 16, Cuadrícula 1; MAQUEDA, 2002, nº 10. 


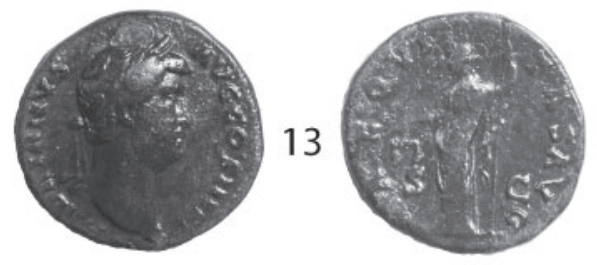

Figura 14: Moneda $\mathrm{n}^{\circ} 13$.

A: HADRIANVS AVG COS III PP. Cabeza laureada a derecha.

R: AEQUITAS. Equidad a izquierda, porta balanza y vara. S - C en campo.

13,32 g; 25 mm; 8 h. Ref. Bibliográfica: RIC II no 795 pág. 441 .

$\mathrm{N}^{\circ}$ Inv. $02 / 79 / \mathrm{C}-1 / 16 / 10$.

14.- Ae, As, Adriano, 117 - 138 d.C.; UE. 16, Cuadrícula 3; MAQUEDA, 2002, nº 14.
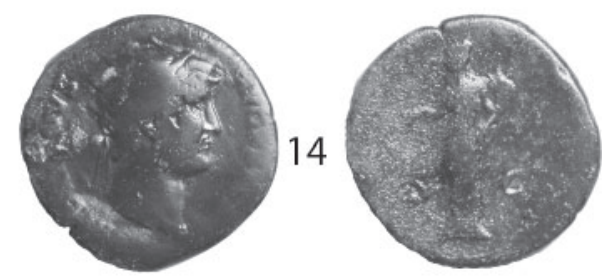

Figura 15: Moneda $\mathrm{n}^{\circ} 14$.

A: Cabeza radiada de Adriano a derecha.

R: Figura femenina a izquierda con cornucopia.

$11,22 \mathrm{~g} ; 27 \mathrm{~mm} ; 6 \mathrm{~h}$.

$\mathrm{N}^{\circ}$ Inv. $02 / 79 / \mathrm{C}-3 / 16 / 14$.

15.- Ae, As, Sabina, Roma, 136 d.C.; UE. 16, Cuadrícula 2; MAQUEDA, 2002, $\mathrm{n}^{\circ} 12$.

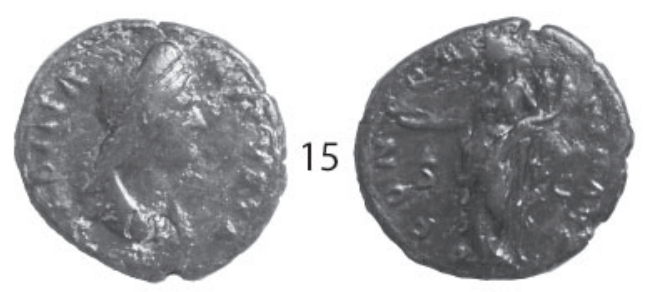

Figura 16: Moneda $n^{\circ} 15$.

A: SABINA AVGVSTA. Busto diademado a derecha con manto y trenza que le llega al cuello.

R: CONCORDIA AVG. Concordia a izquierda, apoyada en columna, porta patena y doble cornucopia. $\mathrm{S}-\mathrm{C}$ en campo.

11,11 g; 29 mm; 6h. Ref. Bibliográfica: RIC II n 1047 pág. 479.

$\mathrm{N}^{\circ}$ Inv. 02/79/C-2/16/12.

16.- Ae, Dupondio, ss. I - II d.C.; 16,21 g; 29 mm; UE. 16, Cuadrícula 2; No Inv. 02/79/C-2/16/1.
17.- Ae, As, ss. I - II d.C.; 8,50 g; 26 mm; UE. 16; No Inv. 02/79/general/16/2A.

18.- Ae, Dupondio, ss. I - II d.C.; 14,45 g; 30 mm; UE. 16, Cuadrícula 5; No Inv. 02/79/C-5/16/2B.

19.- Ae, As, ss. I - II d.C.; 10,45 g; 26 mm; UE. 16, Cuadrícula $1 ; \mathrm{N}^{\circ}$ Inv. 02/79/C-1/16/3.

20.-Ae, As, ss. I - II d.C; 11,39 g; 26 mm; UE. 16; No Inv. 02/79/general/16/2E.

21.- Ae, As, ss. I - II d.C.; 7.86 g; 25 mm; UE. 16, Zanja 2-3; $N^{\circ}$ Inv. 02/79/zanja 2-3/16/1.

22.- Ae, As, ss. I - II d.C.; 9,31g.; 26 mm; UE. 16, Cuadrícula 5; No Inv. 02/79/C-5/16/2A.

23.- Ae, As, ss. I - II d.C.; 8,36 g; 26 mm; UE. 16; $\mathrm{N}^{\circ}$ Inv. 02/79/general/16/2C.

24.- Ae, As, ss. I - II d.C.; 6,83 g; 26 mm; UE. 16; No Inv. 02/79/general/16/2B.

25.- Ae, As, ss. I - II d.C.; 11,50 g; 24 mm; UE. 16; N $^{\circ}$ Inv. $02 / 79 /$ general/16/2D.

\section{VALORACIÓN SOCIO-ECONÓMICA DE LA VILLA A TRAVÉS DE SUS MONEDAS}

Debido al haber encontrado un gran número de elementos que de manera directa e indirecta explican la presencia de este número de monedas en este yacimiento, hemos decidido establecer un comentario conjunto donde los datos históricos de la ciudad se amalgamen con las reflexiones que nos genera el conjunto numismático, por lo que podremos aportar una visión más general de la villa y de sus relaciones con la ciudad de Ávila.

La antigua ciudad de Ávila se encontraba perfectamente comunicada por su cercanía a vías romanas de carácter secundario, las cuales permitían la conexión de este enclave con centros importantes de la provincia lusitana y tarraconense (múltiples enlaces con la vía Augusta Emerita - Asturica Augusta y la vía Augusta Emerita - Caesaragusta) (Abad, 1995; Mariné, 1995, 300-307; Centeno y Quintana, 2005; Fabián, 2007). Esta situación permitió a la ciudad adherirse a los diferentes circuitos comerciales generados por el trazado viario romano.

Las cerámicas, vidrios, vino, aceite, y otras manufacturas no viajaban solas, pues se necesitaba del uso de la moneda para realizar sus respectivas transacciones económicas. Como es evidente, la villa de Huerta de San Nicolás no se mantuvo alejada de dichos circuitos, y más aún al encontrarse en la zona suburbana de la ciudad, como así lo prueban los numerosos fragmentos de terra sigillata hispánica registradas en los dos 
momentos de ocupación, siendo más numerosas en el segundo que en el primero, donde las formas cerámicas arrojan una cronología que va desde la dinastía flavia hasta bien entrado el siglo II d.C. (Romero y Ruiz, 2005, 188-191). Algunos de los sellos documentados por Maqueda, corresponden a los talleres de Trititum Magallum (Tricio, La Rioja), al igual que la mayoría de las cerámicas coetáneas aparecidas en los niveles romanizados de la intervención efectuada en la Plaza de Santa Teresa de Ávila (conocida como Mercado Grande) por Alacet Arqueólogos, S.L. en 2001 (Centeno y Quintana, 2005). Este tipo de materiales llegaba a Ávila y a sus zonas aledañas a través de las vías que hemos citado anteriormente, pues se ha demostrado la importancia de centros como Augusta Emerita y Caesaragusta en la distribución de terra sigillatas (Sáez y Sáez, 1999; Centeno y Quintana, 2005). La cerámica de uso doméstico, en este caso la de engobe negro, es relacionada por Centeno y Quintana (2005) según sus hallazgos en Mercado Grande, con las producciones de los talleres de Lucus Augusti (Alcorta, 1995, 225), las cuales eran comercializadas en la zona noroccidental de la Península Ibérica (Beneítez et alii, 1999), llegando a Ávila a través de los ramales secundarios procedentes de la vía que conectaba Asturica Augusta con Augusta Emerita, mayormente conocida como la vía de la Plata.

Por esto mismo, la moneda encontrada en la villa de Huerta de San Nicolás responde a patrones similares estudiados en el entorno de la vía de la Plata y en las poblaciones vecinas de la zona del Valle del Duero.

La pieza más antigua documentada corresponde con un as del emperador Claudio I, concretamente con el tipo Constantia Augusta (moneda no 1). Teniéndose en cuenta el arte de esta pieza, no sería extraño pensar que pudiéramos encontrarnos ante una pieza de imitación hispana, siendo el tipo Minerva ( $R I C$ I $\mathrm{n}^{\circ} 100$ pág. 127) el más frecuente en el entorno de la Vía de la Plata (Blázquez, 2002, 283). Aun así, no sería insólito que alguna de las monedas frustras fueran en su día ases acuñados a nombre de este emperador, en función de los pesos y módulos que presentan y el acusado desgaste con el que han aparecido. Las monedas de imitación de Claudio son el claro ejemplo de ases de procedencia hispana acuñados ante la necesidad de numerario producido por el cierre de la ceca de Roma (Blázquez, 2002, 283). Acuñaciones que estuvieron circulando en gran número por la vía de la Plata durante el siglo I d.C. e incluso durante el II y III d.C. (Ripollès, 2002, 201) y también con una presencia acusada en las ciudades situadas en la cuenca del Duero (Arias, 2012, 104-105), al igual que en los campamentos militares cercanos, como el caso de Pentavonium donde los hallazgos de ases de Claudio son muy numerosos (Besombes, 2006), patrón común en gran parte de la Península Ibérica (Ripollès, 2002, 201).

La moneda de Nerón (moneda $\mathrm{n}^{\circ} 2$ ) resulta un tanto llamativa al ser el numerario de este emperador escaso en la provincia lusitana (Blázquez, 2002, 286) y en el resto de Hispania (De la Hoz, 2004) pero no tanto en algunas ciudades de la meseta norte (Arias, 2012: 106), existencia que se relaciona con las revueltas de astures que se produjeron durante este reinado (Blázquez, 2002, 284-285) y que expresa un carácter residual en el numerario de esta villa en función de su acusado desgaste, como así también hemos considerado la emitida en el reinado de Claudio I.

Por lo que respecta a la moneda de la dinastía flavia, de las 15 monedas catalogadas, 7 pertenecen a este periodo, 6 (3 dupondios y 3 ases) acuñadas bajo el emperador Vespasiano (monedas $n^{\circ} 3-8$ ) y una con Domiciano (moneda $n^{\circ}$ 9). Las piezas presentan reversos típicos de ambos reinados como la Securitas Augusti (n ${ }^{\circ} 3$, RIC II 744), la Felicitas Publica (n' 4, RIC II 554), la Fortuna Reducis (n 5 RIC II 754a y n ${ }^{\circ}$ 6 RIC II 487) y Pax Augusta (no 7 RIC II 559c) para el reinado de Vespasiano y Saluti Augusti (no 9 RIC II 272) para el de Domiciano; reversos que en su mayoría pretendían difundir entre la población del Imperio la prosperidad y tranquilidad alcanzada después de la guerra civil del 68-69 d.C. (Jacobo, 2003, 223). Sobre estas monedas se observa cierto desgaste, producto de una larga circulación y de la estabilidad política que estaba viviendo el Imperio a finales del siglo I d.C. y principios del siglo II d.C., traducida ésta en una regularidad del aprovisionamiento de moneda (Ripollès, 2002, 204). Los valores documentados son el dupondio y el as, no encontrándonos piezas de plata, que si son frecuentes en la Lusitania y en la zona norte de la meseta (Arias, 2012, 106). No obstante, los hallazgos de ambos emperadores son frecuentes en contextos urbanos localizados en la franja comprendida entre el Tajo y el Duero, la actual Salamanca, relacionando este aspecto Blázquez con la labor política y económica desarrollada por la dinastía flavia en la Lusitania (Blázquez, 2002, 290). Sin embargo, a estos hallazgos deben sumárseles, según la misma autora, los localizados en castros romanizados de la zona del Duero y $\mathrm{NO}$, donde por estas fechas se empieza a consolidar su proceso de urbanización, entendiéndose este también como un aumento económico de signo positivo (Blázquez, 2002, 288; Blázquez, 2005). Hernández Sobrino $(2005,240)$ afirma que es en época flavia cuando la actual ciudad de Ávila alcanza el derecho latino, pero a pesar de ello, la misma autora cree que a diferencia de los demás centros urbanos cercanos, Ávila no fue una ciudad pujante en estos momentos, sino una ciudad ganadera basada en la economía trashumante (Hernández, 2008). Reflexión muy parecida a la que establece Mariné cuando intenta aproximarse a la población que residía en Ávila en el siglo I d.C, quien considera que esta no sería muy numerosa y que estaría dedicada al cultivo de las tierras de alrededor (Mariné, 1995, 306). Por su parte, Fabián (2007) sí identifica desde finales del siglo I d.C. a mediados del siglo II d.C. un gran desarrollo de la ciudad, atendiendo principalmente a las evidencias que han arrojado las múltiples 


\begin{tabular}{|c|c|c|c|c|c|c|}
\hline $\mathbf{N}^{\circ}$ & Emperador & Ceca & Cron. & RIC & UE & Tipos Materiales asociados \\
\hline 1 & Claudio & Roma & 41-54 d.C. & I, 95 & 16 & \multirow{5}{*}{$\begin{array}{l}\text { Estructuras: Elaboradas con aparejo menos } \\
\text { cuidado y con cierta irregularidad. } \\
\text { (Continua) }\end{array}$} \\
\hline 2 & Nerón & Roma & 54-68 d.C. & No cat. & 16 & \\
\hline 3 & Vespasiano & Lugdunum & $72-73$ d.C. & II, 744 & 16 & \\
\hline 4 & Vespasiano & Roma & 74 d.C. & II, 554 & 16 & \\
\hline 5 & Vespasiano & Lugdunum & 77-78 d.C. & II, 754b & 16 & \\
\hline 6 & Vespasiano & Roma & 71 d.C. & II, 487 & 22 & $\begin{array}{l}\text { Estructuras: de grandes dimensiones en opus } \\
\text { incertum } \\
\text { Cerámica: Terra sigillata en fragmentos muy } \\
\text { pequeños y restos de cerámica común engobada de } \\
\text { color negro. }\end{array}$ \\
\hline 7 & Vespasiano & Roma & 74 d.C. & II, 559 & 16 & \multirow{19}{*}{$\begin{array}{l}\text { (Continuación) } \\
\text { Cerámica: de diversos tipos. Terra sigillata con } \\
\text { formas Drag.15/17, Drag. 24/25, Drag. 36, Drag. } \\
\text { 37a, Drag. 29/37 y Drag. 44. También sellos } \\
\text { procedentes de los talleres de Trititum Magallum. } \\
\text { Fragmentos de paredes finas y cerámica } \\
\text { estampillada. } \\
\text { Fragmentos de cerámica común. }\end{array}$} \\
\hline 8 & Vespasiano & ¿? & 69-79 d.C. & No cat. & 16 & \\
\hline 9 & Domiciano & Roma & 85 d.C. & II, 272 & 16 & \\
\hline 10 & Trajano & Roma & 101-102 d.C. & II, 59 & 16 & \\
\hline 11 & Trajano & Roma & 114-117 d.C. & II, 363 & 16 & \\
\hline 12 & Trajano & Roma & 98-99 d.C. & II, 398 & 16 & \\
\hline 13 & Adriano & Roma & 134-138 d.C. & II, 795 & 16 & \\
\hline 14 & Adriano & ¿? & 117-138 d.C. & No cat. & 16 & \\
\hline 15 & Sabina & Roma & 136 d.C. & II, 1047 & 16 & \\
\hline 16 & Frustro & ¿? & ss. I-II d.C. & No cat. & 16 & \\
\hline 17 & Frustro & ¿? & ss. I-II d.C. & No cat. & 16 & \\
\hline 18 & Frustro & ¿? & ss. I-II d.C. & No cat. & 16 & \\
\hline 19 & Frustro & ¿? & ss. I-II d.C. & No cat. & 16 & \\
\hline 20 & Frustro & ¿? & ss. I-II d.C. & No cat. & 16 & \\
\hline 21 & Frustro & ¿? & ss. I-II d.C. & No cat. & 16 & \\
\hline 22 & Frustro & $i ?$ & ss. I-II d.C. & No cat. & 16 & \\
\hline 23 & Frustro & ¿? & ss. I-II d.C. & No cat. & 16 & \\
\hline 24 & Frustro & i? & ss. I-II d.C. & No cat. & 16 & \\
\hline 25 & Frustro & ¿? & ss. I-II d.C. & No cat. & 16 & \\
\hline
\end{tabular}

Figura 17: Cuadro resumen de los hallazgos monetales y arqueológicos.

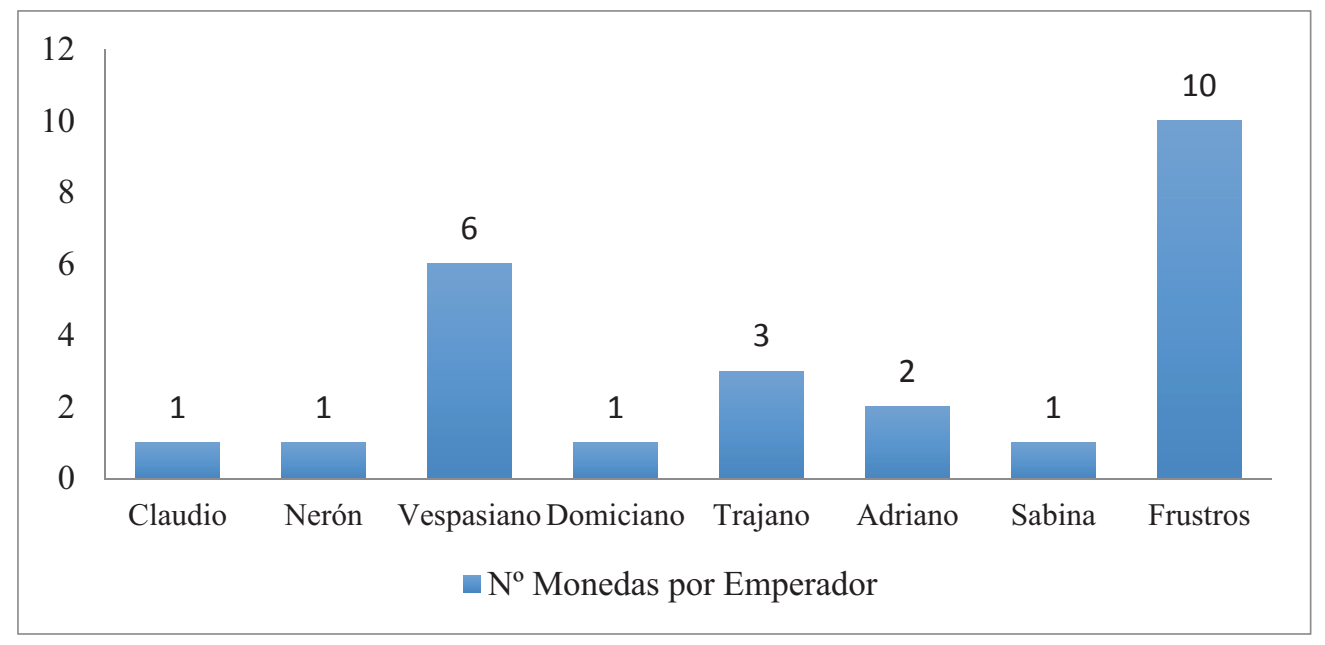

Figura 18: Gráfico resumen donde se indica el número de monedas emitidas por cada emperador. 
intervenciones arqueológicas en el casco urbano. Aún así, atendiendo al registro monetario, no sería de extrañar que nos encontráramos ante la misma dinámica de los castros romanizados estudiados por Blázquez, ya que la villa romana de Huerta de San Nicolás responde al sistema de villae que empiezan a emergen en torno a centros urbanos de la meseta norte, a mediados del siglo I d.C., en el proceso de explotación territorial llevado a cabo por el Imperio Romano en la cuenca del Duero (Blanco et alii, 2009).

Las monedas que cierran el conjunto fueron acuñadas bajo la dinastía Antonina. (2 denarios y 1 dupondio de Trajano; 1 dupondio y 1 as de Adriano y 1 as de Sabina). Una época en la que continua la estabilidad sociopolítica anterior con un desarrollo económico importante (Ripollès, 2002, 205; Blázquez, 2002, 291). Un periodo de perdurabilidad y continuidad bien reflejado en los reversos documentados, victorias $\left(\mathrm{n}^{\circ}\right.$ 10 RIC II 59) y alegorías de la providencia ( $n^{\circ} 11$ RIC II 36b) de la abundancia (n ${ }^{\circ} 12$ RIC II 398), de la equidad (n 13 RIC II 795) y de la concordia (nº15 RIC II 795). A pesar que en la zona de la meseta norte y en el entorno de la vía de la Plata los valores más frecuentes son el sestercio (Arias, 2012, 116; Blázquez, 2002, 292 ), en castros romanizados, villae alto-imperiales y contextos domésticos urbanos, el as sigue estando muy presente al tener aun según Arias Ferrer (2012, 117), cierta importancia en la circulación monetaria de tipo cotidiano. Este mismo periodo de estabilidad sigue coincidiendo con el que se está viviendo en la propia ciudad, según los datos recogidos por Centeno (2006) y Fabián (2007).

Por otra parte, no podemos olvidarnos de dos denarios acuñados bajo mandato del emperador Trajano. Estas dos piezas llaman poderosamente la atención debido a que no solían ser monedas que se perdieran con relativa frecuencia por su valor intrínseco. En la zona de la meseta norte no es muy significativa su presencia en beneficio del bronce como acabamos de mencionar, por el contrario, en las vecinas zonas de Zamora, Blázquez advierte un incremento de la circulación de la plata, aunque sigue predominando el bronce (Blázquez, 2004), aún así, la presencia de este tipo de monedas está relacionada según Arias Ferrer $(2012,114)$ con el mundo militar y las explotaciones mineras, como así sucede en el caso de Pentavonium donde la moneda de plata es muy representativa durante la dinastía antonina (Blázquez y Gómez Barreiro, 2006, 213).

Es evidente que en función de los restos documentados y explicados, la villa de Huerta de San Nicolás acabaría abandonándose a mediados del siglo II d.C., de hecho, podemos comprobar cómo las monedas más recientes documentadas en este yacimiento, correspondientes a la dinastía antonina, no presentan un desgaste acusado, como si se ha podido observar en las precedentes. Por ello, en función de esto y de lo que se ha expresado en páginas anteriores, afirmáramos que la villa presentó en su segundo nivel de ocupación una vida corta pero intensa, pues se puede advertir la presencia de un gran número de transacciones económicas en muy poco tiempo a través de la cerámica documentada y la moneda perdida, no siguiendo así la evolución de las demás villae documentadas en la actual provincia de Ávila, donde las evidencias arqueológicas demuestran la entrada de estas en el siglo III d.C. con la presencia de estructuras arquitectónicas menos cuidadas que aprovechan como cimiento otras anteriores, existencia de terra siguillata tardía y hallazgos frecuentes de Antoninianos, moneda que ejemplifica a la perfección el paulatino deterioro de la economía imperial romana para este siglo (Ripollès, 2002).

\section{CONSIDERACIONES FINALES}

La villa romana de Huerta de San Nicolás debe entenderse como un emplazamiento suburbano, centro agrario y residencial, producto del proceso de explotación territorial llevado a cabo por los romanos en el valle del Duero a mediados del siglo I d.C. (Blanco et alii 2009). Una valoración que puede permitir arrojar más luz a los diferentes problemas existentes en cuanto a la definición de la historia de la Ávila romana.

El registro monetario unido a las evidencias cerámicas nos aportan una imagen bastante interesante de cómo este villa, al igual que la ciudad, se encontraba perfectamente integrada en los diferentes circuitos comerciales gracias, como ya hemos expuesto anteriormente, a la gran cantidad de vías secundarias que rodean el territorio de la ciudad. No obstante, a pesar de este hándicap tan importante de cara al desarrollo comercial y poblacional, la inmensa mayoría de $v i$ llae localizadas en los territorios de la actual provincia de Ávila presentan unos inicios más tardíos. Tan solo existen una serie de yacimientos coetáneos de los cuales solo conocemos moneda más reciente, como el caso de Pared de los Moros en la vecina localidad de Niharra (Abad, 1995). Este hecho hace que tanto el numerario documentado en Huerta de San Nicolás como la villa en sí deba tenerse en cuenta de cara a conocer desde un punto de vista socio - económico el devenir histórico de la ciudad de Ávila.

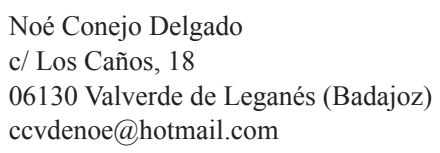

\section{BIBLIOGRAFÍA}

ABAD VARELA, M., 1995: «Aproximación a la economía monetaria en la provincia de Ávila durante la Edad Antigua», en Ma. P. García-Bellido y R. M. Sobral Centeno (eds.), La moneda hispánica. Ciudad y Territorio, I EPNA, 207-214, Madrid.

ALCORTA IROSTARZA, E.J., 1995: «Avance al estudio de la cerámica común romana de cocina y mesa de Lucus 
Augusti», en Cerámica comuna romana d'època Alto Imperial a la Península Ibèrica. Estat de la Cuestió, Monografies Emporitanes VIII, 201-226, Empùries.

ARIAS FERRER, L., 2012: Hispania en el siglo II d.C.: circulación y perduración de la moneda, BAR International Series, Oxford.

BENEÍTEZ, C., HELVIA, S. y MONTES, R., 1999: «Cerámica común romana del Chao Sanmartín (Grandas de Salime, Asturias). I. Vajilla de mesa y despensa», Lancia, 3, 11-48.

BESOMBES, J.C., 2006: «Les monnaies de Claude provenant de camps romains» en $\mathrm{M}^{\mathrm{a}}$. P. García-Bellido (coord.), Los campamentos romanos en Hispania (27 a.C. - 192 d.C.), 557-566, Madrid.

BLANCO GONZÁLEZ, A., LÓPEZ SÁEZ, J.A. y LÓPEZ MERINO, L., 2009: «Ocupación y uso del territorio en el sector centromeridional de la cuenca del cuero entre la Antigüedad y la Alta Edad Media (Siglos I - IX d.C.)», AEspA, 82, 275-300.

BLAZQUEZ CERRATO, $\mathrm{M}^{\mathrm{a}}$ C., 2002: La circulación monetaria en el área occidental de la Península Ibérica. La moneda en torno al Camino de la Plata, Montagnac.

BLÁZQUEZ CERRATO, Mª C., 2004: «La presencia de moneda en la provincia de Zamora: Análisis del poblamiento a través del documento monetal», Zephyrus, 57, 319-366.

BLÁZQUEZ CERRATO, Ma. C., 2005: «Vías de comunicación y circulación monetaria en la Meseta Norte y el Noroeste» en C. Fernandez Ochoa y P. García Díaz (eds.), Unidad y Diversidad en el Arco Atlántico en época romana, III Coloquio Internacional de Arqueología e Gijón, International Series 1371, 235-244, Oxford.

BLÁZQUEZ CERRATO, Mª C., 2006: «Pentavonium (Rosinos de Viriales, Zamora). Circulación monetaria en Pentavonium», en $\mathrm{M}^{\mathrm{a}}$. P. García Bellido (coord.), Los campamentos romanos en Hispania (27 a.C. - 192 d.C.), 203-218, Madrid.

CENTENO CEA, I., 2006: «La imagen de la ciudad alto imperial», en S. Estremera (coord.), Arqueología urbana en Ávila. La intervención en los solares del palacio de Don Gaspar del Águila y Bracamonte, 89-110, Ávila.

CENTENO CEA, I. y QUINTANA LÓPEZ, J., 2005: «Cerámica romana del mercado grande de Ávila. II. Cerámica de mesa de los niveles romanizados», BSAA arqueología, LXXI, 209-273.

DE LA HOZ MONTOYA, J., 2004: «patrones de la distribución de la moneda de bronce de Nerón: II. La distribución regional de los ases», Habis, 35, 257-275.
FABIAN, J.F., 2007: «Los orígenes de la ciudad de Ávila y la época antigua. Aportaciones desde la arqueología al esclarecimiento de las cuestiones históricas previas a la etapa medieval», Ávila en el tiempo. Homenaje al Profesor Ángel Barrio, Vol. I, 83-111, Ávila.

HERNANDO SOBRINO, Ma. R., 2005: Epigrafía romana de Ávila, Ávila.

HERNANDO SOBRINO, Mª R., 2008: «Ávila: una ciudad con vocación ganadera», en J. Mangas Manjarrés y $\mathrm{M}$. A. Novillo (coords.), El territorio de las ciudades romanas, 385-424.

JACOBO PÉREZ, A., 2003: Avtoritas et maiestas: historia, programa dinástico e iconografía en la moneda de Vespasiano, Alicante.

MAQUEDA GARCÍA-MORALES, R., 2002: Informe final de la actuación arqueológica de la zona denominada Huerta de San Nicolás: Plan Parcial Arup 1/1, San Nicolás. (Documento Inédito).

MARINÉ, M., 1995: «La época romana», en M. Mariné (coord.), Historia de Ávila. I. Prehistoria e Historia Antigua, 282-338, Ávila.

PÉREZ RODRÍGUEZ-ARAGÓN, F., 2014: «Los centros de producción de la terra sigillata hispánica tardía. Antiguos y nuevos centros, hornos y estructuras asociadas», Oppidum, 10, 147-176.

ROMERO CARNICERO, Mª . V. y RUIZ MOTES, P., 2005: «Los centros de producción de TSH en la zona septentrional de la Península Ibérica», en M. Roca Roumens y $\mathrm{M}^{\mathrm{a}}$. I. Fernández García (coords.), Terra Sigillata Hispánica. Centros de fabricación y producción alto imperiales. Homenaje a $M^{a}$. A. Mezquíriz, 184-223, Jaén-Málaga.

RIC (Roman Imperial Coinage): t. I: SUTHERLAND, C.H.V., 1984: Roman Imperial Coinage, from Augustus to Vitellius, London. t. II: MATTINGLY, H.-SYDENHAM, E. A., 1926: Roman Imperial Coinage, from Vespasian to Hadrian, London.

RIPOLLÈS, P.P., 2002: «La moneda romana imperial y su circulación en Hispania», AEspA, 75, 195-214.

SÁEZ PRECIADO Ma . P. y SÁEZ PRECIADO, C., 1999: «Estado de la cuestión de las alfares riojanos. La terra sigillata hispánica altoimperial», en M. Roca Roumens y Mª I. Fernández García (coords.), Terra Sigillata Hispánica. Centros de fabricación y producción alto imperiales. Homenaje a $M^{a}$. A. Mezquíriz, 61-136, Jaén-Málaga.

Recepción: 20-05-2015

Aceptación: 13-07-2015 